was each derived from two or three unrelated donors. The primary outcome was clinical remission with Mayo score.

Results 20 patients were enrolled in our trial, of whom all received capsules. The primary outcome with clinical remission was achieved in 15 out of 20 patients (75\%), and 5 out of 20 patients $(25 \%)$ had a relapse. No adverse events were identified in these patients. The quantity of lipopolysaccharide was higher in patients with ulcerative colitis compared to normal subjects, but no obvious change was identified between responders and non-responders patients after faecal microbiota transplantation. What's more, increased expression of CD5 $+\mathrm{CD} 19+\mathrm{B}$ cell, CD3-CD16+CD56+NK cell, and CD4 $+\mathrm{CD} 25+$ Foxp3+Treg cell was found after FMT, which indicated that FMT by capsules could induce an anti-inflammatory pathway in patients with active ulcerative colitis. This may regenerate immunological tolerance to defence the increased inflammation by endotoxin.

Conclusions Intensive-dosing, multidonor, FMT by capsules induces clinical remission in active ulcerative colitis and is associated with the changes of the immune system. FMT by capsules is, thus, a promising new therapeutic option for ulcerative colitis with more economical and convenient. Future work should focus on the multicenter verification and the relationship of immunity and gut microbiome.

\section{IDDF2018-ABS-0234 AN ANALYSIS OF TREND, CLINICO- PATHOLOGICAL FEATURES AND TREATMENT RECEIVED BY MULTI-ETHNIC PATIENTS WITH COLORECTAL CANCER AT A SINGLE TERTIARY CARE CENTRE IN MALAYSIA: A REVIEW OF 2 DECADES}

'Zhiqin Wong*, 'Zairul Azwan Mohd Azman, 'Luqman Mazlan, 'Rasyidah Rehir, 'Chai Soon Ngiu, ${ }^{1}$ Jeevinesh Naidu, ${ }^{3}$ Fuad Ismail, ${ }^{3}$ Nik Muhd Aslan Abdullah, ${ }^{1}$ Gee Huey Leong, ${ }^{4}$ Isa Rose, ${ }^{4}$ Azyani Yahaya, ${ }^{5}$ Nur Yazmin Yaacob, ${ }^{5}$ Syazarina Sharis Osman, ${ }^{2}$ Ismail Sagap, ${ }^{1}$ Raja Affendi Raja Ali. 'Gastroenterology Unit, Department of Medicine, Faculty of Medicine, UKM Medical Centre, Malaysia; ${ }^{2}$ Colorectal Unit, Department of Surgery, Faculty of Medicine, UKM Medical Centre, Malaysia; ${ }^{3}$ Department of Oncology, Faculty of Medicine, UKM Medical Centre, Malaysia; ${ }^{4}$ Department of Pathology, Faculty of Medicine, UKM Medical Centre, Malaysia; ${ }^{5}$ Department of Radiology, Faculty of Medicine, UKM Medical Centre, Malaysia

\subsection{6/gutjnl-2018-IDDFabstracts. 167}

Background The incidence of colorectal cancer (CRC) is increasing steadily in Asia Pacific region including Malaysia. The collaborative data collection system enables CRC registration to document details of patients with CRC. This study aim to analyse the trend, clinico-pathological features along with treatment modalities received by CRC patients at our centre from 1997-2017.

Methods A Total of 1000 CRC cases were analysed from the hospital medical record system.

Results The median age at diagnosis was $63+11.97$ years. Majority of patients diagnosed between 50 to 69 years old $(57.9 \%)$ and $60.5 \%$ were males. The trend of CRC among the young patients revealed; Age group 20-39 years at 5.1\%, and age group 40-49 years at $10.8 \%$. There was an increment of $14.3 \%$ CRC cases in the second decade of analysis. Chinese patients were highest at $52.9 \%$, followed by Malays at $42.3 \%$ and Indians at $3.1 \%$. However, for young patients
( $<50$ years old), Malays predominate at 55.3\%, and for elderly (>70 years old), Chinese predominate at 59.9\%. Most CRC cases were in the rectum $(34.1 \%)$ with the majority of adenocarcinoma (83.3\%) of well-differentiated cells (44.4\%). For patients with Duke A:65.4\% had surgery alone and $15.4 \%$ had surgery, chemotherapy and radiotherapy. For patients with Duke B (34.1\%) and C (39.2\%): 40.9\% had surgery alone, $26.6 \%$ had surgery and chemotherapy and $15.1 \%$ had surgery and chemo-radiotherapy. For patients with Duke D; 33.3\% had palliative surgery along and 9.1\% had surgery and chemo-radiotherapy. Anterior resections were most frequently performed at 38\%.

Conclusions In Malaysia, most CRC cases were diagnosed in the rectum at Dukes $\mathrm{B}$ and $\mathrm{C}$, which commonly occurred among middle-aged and elderly Chinese patients. Majority of CRC cases in the young $(<50$ years old $)$ patients were Malays. Most of the patients received only surgical treatment with anterior resections most frequently performed. There is a definite increasing trend of CRC in particular among young Malay patients for the past 2 decades in Malaysia.

\section{IDDF2018-ABS-0236 ABDOMINAL COCOON SYNDROME: A CASE REPORT AND REVIEW OF RELATED LITERATURE}

Michael Arrosas*. University of the East Ramon Magsaysay Memorial Medical Center- GIT Unit, Philippines

\subsection{6/gutjnl-2018-IDDFabstracts. 168}

Significance Despite several cases reported worldwide, Abdominal Cocoon Syndrome still is a rare condition. No published reports have been found locally. And reminds us to always be prudent despite being presented with a common problem. The correct early diagnosis avoids the risk of complications. This report aims to keep us abreast of the current practices and recommendation by other countries and able to be better equipped in handling perioperative incidental findings of this entity prior to the end of surgery.

Clinical Presentation We presented a case of a 30-year-old man subacute gastric outlet obstruction and lagged physical development not appropriate for age.

Management Imaging revealed dilated stomach and duodenum, delayed barium transit from the stomach to ileum, and concomitant distal jejunal and proximal ileal obstruction. Initially treated as SMA syndrome, but after 2 attempts of conservative management failed, a diagnostic laparotomy was done. A surprising intraoperative findings revealed a thick membrane covering the entire small intestines sparing the duodenum. Adhesiolysis and removal of the membrane were made, releasing the trapped intestines. Histopathology confirmed the diagnosis. The patient was discharged 2 weeks post-operatively.

Recommendation It is recommended to remain prudent in handling the common problem and always have a high index of suspicion. Maximise preoperative condition of the patient, decide when to do the definitive treatment, careful surgery, judicious postoperative care, and surveillance of possible complications is a must. Aetiology remains elusive, research on the relationship of prematurity at birth, smoking on the 